\title{
Surface-Based Regional Homogeneity in First-Episode, Drug-Naïve Major Depression: A Resting-State fMRI Study
}

\author{
Hui-Jie Li, ${ }^{1}$ Xiao-Hua Cao, ${ }^{2}$ Xing-Ting Zhu, ${ }^{1,3}$ Ai-Xia Zhang, ${ }^{2}$ Xiao-Hui Hou, ${ }^{1,3}$ \\ Yong $\mathrm{Xu}^{2}{ }^{2} \mathrm{Xi}$-Nian Zuo, ${ }^{1}$ and Ke-Rang Zhang ${ }^{2}$ \\ ${ }^{1}$ Laboratory for Functional Connectome and Development, Magnetic Resonance Imaging Research Center, \\ Key Laboratory of Behavioral Science, Institute of Psychology, Chinese Academy of Sciences, No. 16 Lincui Road, \\ Chaoyang District, Beijing 100101, China \\ ${ }^{2}$ Department of Psychiatry, First Hospital of Shanxi Medical University, No. 85 Jiefang South Road, Taiyuan 030001, China \\ ${ }^{3}$ University of Chinese Academy of Sciences, Beijing 100049, China
}

Correspondence should be addressed to Xi-Nian Zuo; zuoxn@psych.ac.cn and Ke-Rang Zhang; krzhang_sxmu@vip.163.com

Received 5 November 2013; Revised 15 January 2014; Accepted 19 January 2014; Published 25 February 2014

Academic Editor: Qiyong Gong

Copyright (C) 2014 Hui-Jie Li et al. This is an open access article distributed under the Creative Commons Attribution License, which permits unrestricted use, distribution, and reproduction in any medium, provided the original work is properly cited.

Background. Previous volume-based regional homogeneity (ReHo) studies neglected the intersubject variability in cortical folding patterns. Recently, surface-based ReHo was developed to reduce the intersubject variability and to increase statistical power. The present study used this novel surface-based ReHo approach to explore the brain functional activity differences between firstepisode, drug-naïve MDD patients and healthy controls. Methods. Thirty-three first-episode, drug-naïve MDD patients and 32 healthy controls participated in structural and resting-state fMRI scans. MDD patients were rated with a 17-item Hamilton Rating Scale for Depression prior to the scan. Results. In comparison with the healthy controls, MDD patients showed reduced surfacebased ReHo in the left insula. There was no increase in surface-based ReHo in MDD patients. The surface-based ReHo value in the left insula was not significantly correlated with the clinical information or the depressive scores in the MDD group. Conclusions. The decreased surface-based ReHo in the left insula in MDD may lead to the abnormal top-down cortical-limbic regulation of emotional and cognitive information. The surface-based ReHo may be a useful index to explore the pathophysiological mechanism of MDD.

\section{Introduction}

Major depressive disorder (MDD) is characterized by cognitive deficits, functional disability, and pervasive negative feelings, such as sadness, anxiety, dysphoria, anhedonia, sleep abnormalities, and feelings of worthlessness [1]. MDD patients have deficits in emotional self-regulation and are prone to negative emotion bias toward life events [2]. Researchers have used behavioral, neurochemical, and electrophysiological approaches to explore the pathophysiology of depression, but the mechanisms of pathophysiology are still unclear.

Most recently, resting-state fMRI (RFMRI) has been widely used to study the functional brain abnormalities of MDD, given the highly synchronous nature of spontaneous low-frequency oscillations $(0.01-0.08 \mathrm{~Hz})$ in motor cortices
[3]. Of the many RFMRI approaches, regional homogeneity ( $\mathrm{ReHo}$ ) has attracted substantial attention in research studies. ReHo employs Kendall's coefficient of concordance (KCC) to measure the functional coherence of a given voxel with its nearest voxels in a voxelwise analysis; it assumes that the spontaneous neural activity of a given voxel is similar to its neighbors [4-6]. ReHo reflects the temporal homogeneity of the regional blood oxygen level dependent (BOLD) signal. Thus, an abnormal ReHo may be related to changes in temporal spontaneous neural activity of a certain region [4].

Various studies explored spontaneous brain activity in MDD with the volume-based ReHo approach. The MDD group included medicated and unmedicated patients and showed decreased ReHo mainly in the frontal and limbic lobes and the basal ganglia [7]. Given that the MDD group included some medicated patients, the results could not 
exclude a potential medication effect. Two other studies explored the ReHo in treatment-resistant depression (TRD) patients and found that TRD patients showed lower ReHo in the left insula, the superior temporal gyrus, the inferior frontal gyrus, the posterior fusiform gyrus, the superior parietal lobule, and the precuneus $[8,9]$. In these two studies, all of the patients were medicated and treatment-resistant; thus, the medication may have contributed to the results. Our previous study investigated the ReHo in 15 first-episode, drug-naïve MDD patients, 15 first-degree relatives, and 15 healthy controls; the results revealed that both MDD patients and the first-degree relatives showed decreased ReHo in the right insula and the left cerebellum [10]. Two other studies investigated first-episode and drug-naïve MDD patients and reported decreased ReHo in the left thalamus, the left temporal lobe, the left cerebellar posterior lobe, and the bilateral occipital lobe [11] and increased ReHo in the fusiform gyrus and the bilateral superior frontal gyrus in comparison with healthy controls [12].

These previous ReHo studies on MDD are based on the 3-dimensional volume-based calculation. Although volumebased activation maps have been used widely to date, this approach neglected the intersubject variability in cortical folding patterns. Therefore, some anatomical regions could not accurately match the same location in the standard template. While under the cortical surface space, the anatomical regions could occupy the same place, reduce the intersubject variability, and increase statistical power [13-15]. Recently, a volume-based ReHo computation was transplanted into a 2-dimensional variant with cortical surface-based fMRI analysis; the surface-based ReHo was found to have moderate to high intersession and intrasession test-retest reliability [16].

In the present study, using this relatively new surfacebased ReHo approach, we aimed to explore whether the surface-based ReHo could be an effective biomarker of MDD. We recruited first-episode and drug-naïve patients to exclude a potential medication effect. Moreover, we also aimed to investigate the relationships between the $\mathrm{ReHo}$ and the clinical variables in the MDD group.

\section{Methods}

2.1. Participants. Thirty-two patients with MDD from 17 to 60 years of age were recruited from the inpatient and outpatient units in the Department of Psychiatry, First Hospital of Shanxi Medical University. All of the patients were diagnosed with a Chinese version of the Modified Structured Clinical Interview for DSM-IV for patient version (SCID-I/P) [17] by two trained clinical psychiatrists. All MDD patients were drug-naïve and in their first episode of illness (the first time they experienced a depressive episode). These patients had no history of other major psychiatric illness, neurological illness, head injury, and alcohol or drug abuse. MDD patients were rated with a 17-item Hamilton Rating Scale for Depression (HRSD) before the imaging scan.

Thirty-three age-, gender-, and education-matched healthy controls were recruited by advertisements. They were interviewed with the Structured Clinical Interview for DSM-IV for nonpatient version (SCID-I/NP). All healthy controls were free of depression or other psychiatric or neurological illness and had no history of head injury and alcohol or drug abuse.

All MDD patients and healthy controls were righthanded. Written informed consent was obtained from each participant. The Medical Research Ethics Committee of the First Hospital of Shanxi Medical University approved this study.

2.2. Data Acquisition. Participants were scanned by the 3.0T Siemens Trio scanner. During the scanning, participants laid supine in the scanner with their heads fixed with foam pads to decrease head motion and reduce scanner noise. They were informed to close their eyes and remain awake while moving as little as possible. The scanning sessions included the following: (i) three-dimensional T1-weighted whole-brain images: 3D-MPRAGE sequence, TR/TE $=2300 / 2.95 \mathrm{~ms}, 160$ slices, thickness $/$ gap $=1.2 / 0.6 \mathrm{~mm}, \mathrm{FOV}=226 \times 240 \mathrm{~mm}$, matrix $=240 \times 256$, and flip angle $=9^{\circ}$ and (ii) the restingstate $\mathrm{fMRI}$ image: echo planar imaging (EPI) pulse sequence, 32 slices, $\mathrm{TR} / \mathrm{TE}=2000 / 30 \mathrm{~ms}$, thickness $/$ gap $=3 / 1 \mathrm{~mm}$, matrix $=64 \times 64, \mathrm{FOV}=240 \times 240 \mathrm{~mm}$, flip angle $=90^{\circ}$, and 212 volumes.

2.3. Data Preprocessing. The Connectome Computation System (CCS: http://lfcd.psych.ac.cn/ccs.html) carries all steps of preprocessing both the structural and functional image preprocessing [16]. The structural image processing included the following steps: (1) the MR image denoise with a spatially adaptive nonlocal means filter [18, 19], (2) reconstruction of cortical surface, (3) segmentation of the cerebrospinal fluid (CSF), white matter (WM), and gray matter (GM) volumetric structures, (4) estimation of a triangular mesh tessellation over the GM-WM boundary and the mesh deformation to produce a smooth representation of the GM-WM interface (white surface) and the GM-CSF interface (pial surface) spatial normalization from individual native space to fsaverage stereotaxic space, (5) correction of topological defect on the surface, (6) inflation of individual surface mesh into a sphere, and (7) estimation of the deformation between the resulting spherical mesh and a common spherical coordinate system. The functional image preprocessing included (1) drop of the first five volumes, (2) slice timing correction, (3) 3D motion correction, (4) 4D global mean-based intensity normalization (mean intensity: 10,000), (5) nuisance regression (the $\mathrm{WM}$ and CSF mean time series and the Friston-24 motion time series) [20], (6) band-pass filtering $(0.01-0.1 \mathrm{~Hz}),(7)$ removal of linear and quadratic trends, (8) coregistration between individual structural and functional images by the GM-WM boundary-based registration (BBR) algorithm [21], and (9) projection of the individual preprocessed 4D RFMRI time series onto a standard cortical surface fsaverage 5 .

Following the preprocessing, a data quality control procedure (QCP) was conducted. The QCP of structural images included visual head motion inspection, tissue segmentation, and pial and white surface reconstruction. 
The QCP of functional images included steps of checking of the minimal cost of coregistration (mcBBR) and the root mean square of framewise displacement (rmsFD) http://lfcd.psych.ac.cn/ccs/QC.html.

2.4. Surface-Based ReHo Analysis. Surface-based ReHo analysis was performed with the CCS. Details of the analysis can be found in our recent study [16]. The individual preprocessed R-FMRI data was projected into the $f$ saverage 5 (FREESURFER 5.1) surface space. For each vertex of the surface space, the corresponding coordinates were computed in anatomical and functional space, and then the trilinear interpolation was used to interpolate the fMRI values [16]. Surface-based ReHo was calculated by Kendall's coefficient of concordance of the time series of a given vertex with those of its nearest neighbors. This computational procedure was repeated for all vertices in surfaces of both hemispheres to produce vertexwise KCC-ReHo surface maps, which are denoted 2-dimensional ReHo (2dReHo). When 7 nearest neighbors were used to explore the $2 \mathrm{dReHo}$, we call it 2dReHo. Moreover, we also used a broader range of 19 neighbors to calculate $2 \mathrm{dReHo}$ and named it $2 \mathrm{dReHo} 2$. The individual $2 \mathrm{dReHo}$ maps were smoothed with a Gaussian kernel of $6 \mathrm{~mm}$ full-width half-maximum for subsequent statistical analyses.

2.5. Statistical Analysis. Unpaired two-sample $t$-tests were performed to compare age and years of education. A chisquare test was used to compare the gender ratio between the two groups. Voxelwise one-way ANCOVA tests (covariates: age, sex, years of education, $\mathrm{mcBBR}$, and rmsFD) were performed to explore the $2 \mathrm{dReHo}$ differences between the MDD and healthy controls. The Pearson correlation coefficients between each vertex on the surface and demographic and clinical variables were calculated. We employed a cluster-level familywise error (FWE) correction for multiple comparisons [22]. Specifically, given a vertexwise statistical parameter map, a vertexwise $P$ value (i.e., uncorrected $P<0.01$ ) was first assigned to form clusters across the entire hemisphere. Based upon the distribution of spatial extents of the survived clusters, an implicit cluster size or extent was generated to achieve the final corrected $P$ value (here, $P<0.05$ ) for controlling the FWE.

\section{Results}

3.1. Participants. Participants' characteristics are shown in Table 1. The two groups were matched by age, gender ratio, and years of education. Moreover, age at illness onset, illness duration, and HRSD score of MDD patients are also included in Table 1.

3.2. Surface-Based ReHo Differences between MDD and Healthy Controls. Across the cortical surface, no significant differences were found between the MDD patients and healthy controls for the global mean ReHo or ReHo2 (Table 1). Moreover, MDD patients had a significant decrease in $\mathrm{ReHo}$ and $\mathrm{ReHo} 2$ in the left insula when compared with healthy controls (Figure 1). There was no increase in ReHo or ReHo2 in MDD patients.

3.3. Correlation Analysis. For MDD group, there were no significant correlations between age/years of education with left insula ReHo values (age: $P=0.26$ and $P=0.26$ for ReHo and ReHo2; years of education: $P=0.81$ and $P=$ 0.68 for ReHo and ReHo2, resp.). Moreover, no significant correlations were found between the left insula ReHo values and the patients' age at illness onset $(P=0.24$ and $P=0.25)$, illness duration $(P=0.90$ and $P=0.99)$, or the HRSD total score $(P=0.41$ and $P=0.43)$.

We also calculated the correlations between global ReHo values and the age and years of education. For MDD group, no significant correlations were found between age/yeas of education and global ReHo values (age: $P=0.36$ and $P=$ 0.44 for ReHo and ReHo2; years of education: $P=0.43$ and $P=0.40$ for ReHo and ReHo2). For healthy control group, there were also no significant correlations between age/years of education and global ReHo values (age: $P=0.25, P=0.29$ for ReHo and ReHo2; years of education: $P=0.72, P=0.72$ for $\mathrm{ReHo}$ and $\mathrm{ReHo} 2$ ).

\section{Discussion}

To the best of our knowledge, this is the first study of surface-based ReHo in MDD. The present results reveal that MDD patients show decreased surface-based ReHo in the left insula; no significant correlations were found between the surface-based ReHo value in the left insula and the clinical characteristics of MDD.

The present surface-based ReHo results are more concise and partly supported by previous volume-based ReHo studies in MDD $[8,10]$. Our previous volume-based ReHo study found that first-episode, drug-naïve MDD patients had decreased volume-based ReHo in the right insula cortex [10]. Different approaches may be the main reason for the differences across studies. Moreover, the participants and the scanning parameters were also different. The present results are supported by a recent meta-analysis of restingstate brain activity in MDD [23]. This meta-analysis included resting-state fMRI studies analyzed by volume-based ReHo and independent component analysis, as well as several PET studies of MDD; the results revealed that MDD patients had lower brain activity in left insula in comparison with healthy controls [23].

Located among the frontal, temporal, and parietal lobe and limbic regions, insula cortex is considered as an integration center of external events and internal cognitive processing $[24,25]$ and plays important role in various emotional and cognitive functions [26]. Compared with healthy controls, MDD patients had decreased activation in the left insula in a series of emotion-related tasks [2729]. Recent RFMRI studies also reported decreased functional connectivity between the left insula and the bilateral amygdala [30] and the insula cortex and the subgenual anterior cingulate cortex [31] in MDD patients. Another RFMRI study further revealed that nonrefractory depression 
TABLE 1: Participants information.

\begin{tabular}{lccc}
\hline & MDD $(N=33)$ & Health control $(N=32)$ & $P$ \\
\hline Age (years) & $34.18(10.96)$ & $34.56(9.92)$ & $13 / 19$ \\
Gender (male/female) & $13 / 20$ & $13.72(2.93)$ & $0.88^{\mathrm{a}}$ \\
Yeas of education & $13.18(3.09)$ & & \\
Illness onset age & $33.91(10.92)$ & & \\
Illness duration (month) & $6.02(6.25)$ & $0.42^{\mathrm{b}}$ \\
HRSD & $20.16(3.22)$ & 0.60 & 0.14 \\
mcBBR & 0.59 & 0.66 & $0.88^{\mathrm{a}}$ \\
rmsFD (mm) & 0.14 & 0.52 & $0.55^{\mathrm{a}}$ \\
Global ReHo & 0.67 & $0.51^{\mathrm{a}}$ \\
Global ReHo2 & 0.52 & $0.63^{\mathrm{a}}$ \\
\hline
\end{tabular}

MDD: major depressive disorder; HRSD: Hamilton Rating Scale for Depression. The values in brackets are standard deviations.

${ }^{a}$ Obtained by two-sample $t$-test. ${ }^{\mathrm{b}}$ Obtained by chi-square test; ${ }^{\mathrm{c}} \mathrm{rmsFD}$ is the root mean square of the framewise displacement for in-scanner head motion.
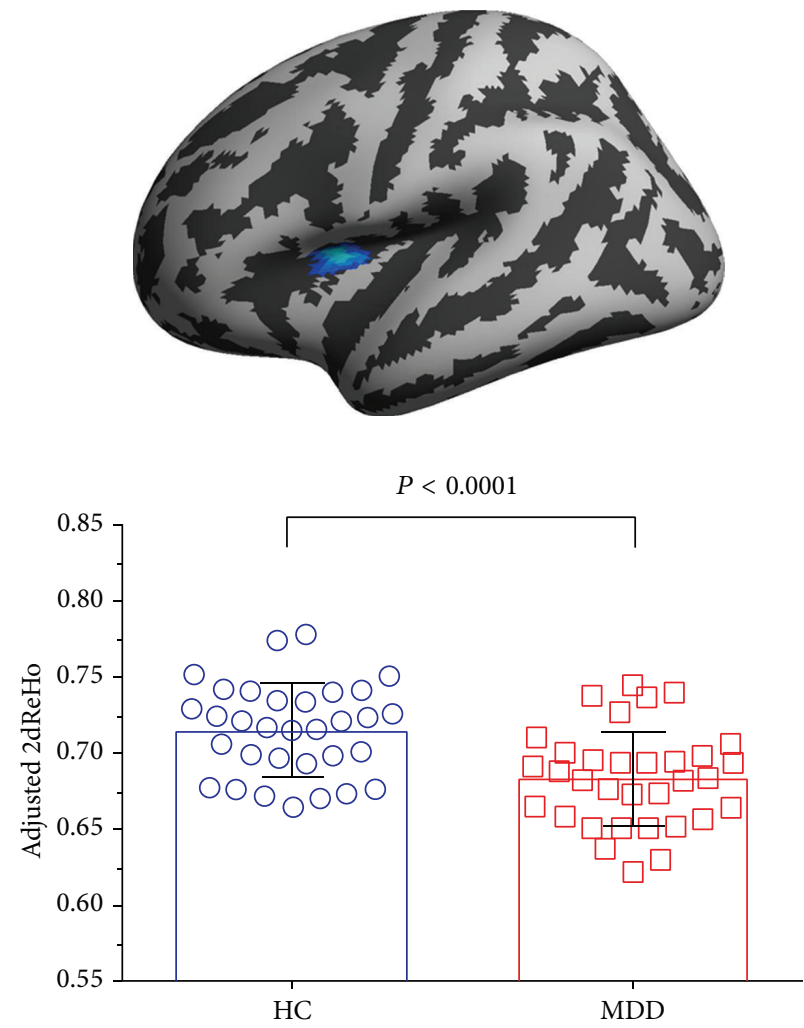

(a)
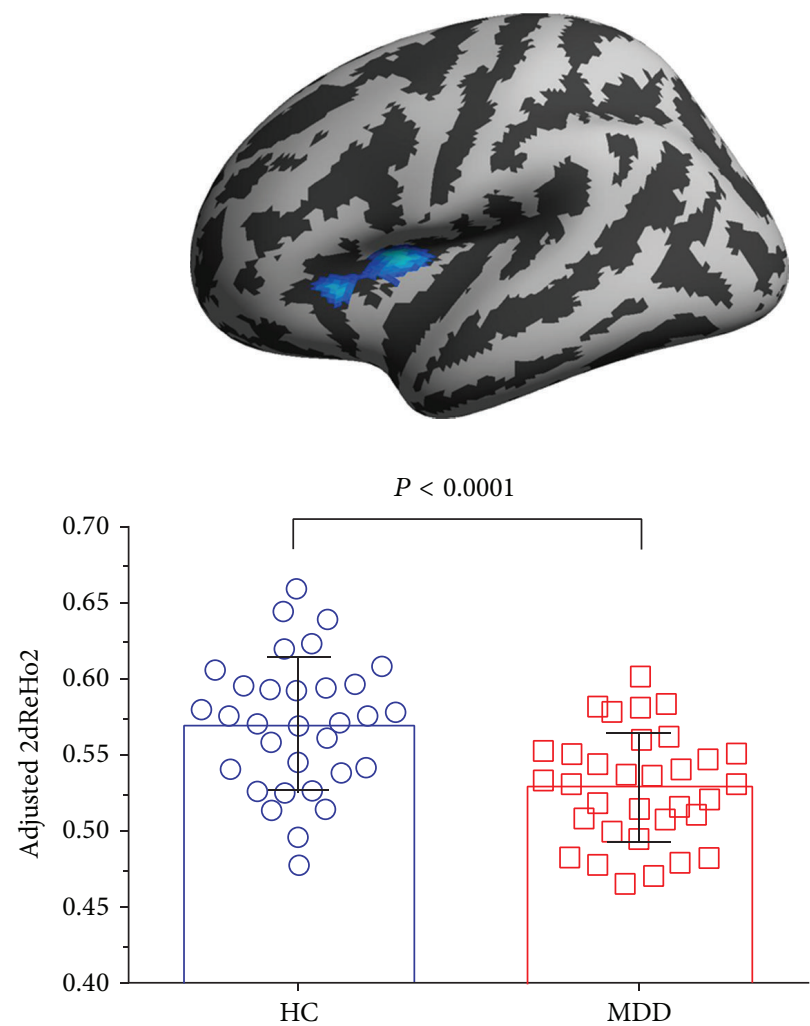

(b)

FIGURE 1: MDD patients showed lower vertexwise functional homogeneity in left insula ((a) 2dReHo and (b) 2dReHo2) compared to healthy controls.

was associated with decreased functional connectivity in the limbic-striatal-pallidal-thalamic circuits, of which the insula is an important part [32]. A recent meta-analysis focusing on cortical-subcortical interactions in emotion demonstrated that the insula is activated across all basic and social emotions [33]. Two other reviews also highlighted the insula as an important region involved in emotional processing and the affective symptoms in MDD [34, 35]. Following a systematic review of the role of the insula cortex in MDD, researchers proposed that, due to the neuroanatomical connections, the insula might also be part of the frontolimbic network [36]; this network plays a very important role in emotion regulation. The decreased activity in the insula cortex of MDD may lead to somatic complaints, emotional dysfunctions, and the negative bias in explaining life events [31]. The abnormal activity of the corticolimbic network leads to the disruptions of top-down processing, which is thought to mediate pervasive emotions of sadness and negative affect in MDD patients.

Recent neuroanatomical and neurochemical studies reveal that the insula cortex plays an important role in the pathophysiology of MDD. MDD patients show significantly 
reduced volume [37] and cortical thickness [38] in the insula cortex. A voxel-based morphometry meta-analysis supports evidence that the clinical high-risk populations for psychosis show reduced gray matter in the left insula [39]. MDD patients also show lower fractional anisotropy in the left insula compared to healthy controls [40]. Several positron emission tomography (PET) studies found that MDD patients showed increased 5-HTT binding potential [41], decreased binding of the metabotropic glutamate receptor [42], and lower rCBF levels [43] in the insula cortex compared to healthy controls.

The correlation analysis shows that the surface-based ReHo value in the left insula cortex was not significantly correlated with the clinical variables and the depressive symptoms. Of the previous volume-based ReHo studies in MDD, only one study reported correlations between the ReHo value in the right insula and the severity of anxiety [7]; several other studies did not find significant correlations between the ReHo values and the clinical variables, as well as the HRSD score in first-episode and drug-naïve MDD [10], early-onset and late-onset drug-naïve MDD [12], TRD [8], and late-life depression [44]. The structural imaging studies also provided inconsistent results. No significant correlations were found between insula volume and the severity of depressive symptoms [37], while significant correlations were reported between the insula cortex and the Beck Depression Inventory, the Hamilton Depression Rating Scale, and the Snaith-Hamilton Pleasure Scale in MDD patients [45]. Together with these correlation results, the correlations between the ReHo values in the potential biomarker regions and the depressive symptoms still require further exploration.

Although the present study focused on the surface-based $\mathrm{ReHo}$, we also analyzed the volume-based ReHo in the same cohort of participants. With the same covariates (age, sex, years of education, $\mathrm{mcBBR}$, and $\mathrm{rmsFD}$ ) and the same statistical criteria (corrected, $Z=2.3, P<0.05$ ), we found no significant clusters between MDD and healthy controls with volume-based ReHo approach. Some technical and methodological reasons may result in the differences between surface- and volume-based ReHo approaches. Firstly, the volume-based ReHo measures local signal synchronization in both gray and white matter, while the surface-based ReHo measures mostly the gray matter local synchronization signals. Therefore, the volume-based ReHo may include some artificial signals from white matter. Secondly, for $2 \mathrm{dReHo}$ and $3 \mathrm{dReHo}$, although we try to keep the same length of neighbors for a certain vertices or voxel, the number of neighbors is different; the $2 \mathrm{dReHol}$ and $2 \mathrm{dReHo} 2$ calculation recruits 6 and 19 neighbors, respectively, while the $3 \mathrm{dReHo}$ recruits 26 neighbors. Moreover, the mask templates are different (MNI $1523 \mathrm{~mm}$ standard volume for 3dReHo, while fsaverage $54 \mathrm{~mm}$ resolution standard space for $2 \mathrm{dReHo}$ ). These factors may influence the results in some degree. Thirdly, on the cortical surface, the intersubject variability in anatomy and the intersubject registration in both brain structure and function may be estimated more accurately [16]. Finally, previous volume-based neuroimaging studies consider that some subcortical regions (amygdala and thalamus) play important role in emotional circuit [46, 47]; however, the present surface-based ReHo only investigates the cortical regions; therefore it cannot reveal the differences in subcortical regions between healthy controls and MDD.

Although the present surface-based ReHo revealed interesting results, this study also had several limitations. First, previous findings suppose that MDD patients show deficits in the prefrontal-amygdala-pallidostriatalmediothalamic mood regulating circuit $[46,47]$. Given that the present surface-based ReHo approach focuses only on the cortical cortex, we did not examine the surface-based ReHo in the subcortical cortex, which are important regions of the mood regulating circuit. Second, the present study did not include neuropsychological tasks, specifically, the emotion processing related tasks. Including these tasks may help to determine whether the first-episode, drug-naïve MDD patients show deficits in the corresponding behavioral levels.

In conclusion, the present study revealed decreased surface-based ReHo in the left insula in MDD patients. The abnormal activity in the left insula may lead to the abnormal top-down cortical-limbic regulation of the emotional and cognitive based information. Moreover, no significant correlations were found between the surface-based ReHo value in the left insula and the age, years of education, age of illness onset, illness duration, and HRSD score in MDD patients.

\section{Conflict of Interests}

The authors declare that there is no conflict of interests regarding the publication of this paper.

\section{Acknowledgments}

This work was supported by the Natural Science Foundation of China (31000465, 81171409, 81000583, 30971054, and 81171290) and the Hundred Talents Program and the Key Research Program (KSZD-EW-TZ-002, XNZ) of Chinese Academy of Sciences.

\section{References}

[1] American Psychiatric Association, Diagnostic and Statistical Manual of Mental Disorders, American Psychiatric Press, Washington, DC, USA, 4th edition, 2000.

[2] G. J. Siegle, S. R. Steinhauer, M. E. Thase, V. A. Stenger, and C. S. Carter, "Can't shake that feeling: event-related fMRI assessment of sustained amygdala activity in response to emotional information in depressed individuals," Biological Psychiatry, vol. 51, no. 9, pp. 693-707, 2002.

[3] B. Biswal, F. Z. Yetkin, V. M. Haughton, and J. S. Hyde, "Functional connectivity in the motor cortex of resting human brain using echo-planar MRI," Magnetic Resonance in Medicine, vol. 34, no. 4, pp. 537-541, 1995.

[4] Y. Zang, T. Jiang, Y. Lu, Y. He, and L. Tian, "Regional homogeneity approach to fMRI data analysis," NeuroImage, vol. 22, no. 1, pp. 394-400, 2004.

[5] Y. Liu, K. Wang, C. YU et al., "Regional homogeneity, functional connectivity and imaging markers of Alzheimer's disease: a review of resting-state fMRI studies," Neuropsychologia, vol. 46, no. 6, pp. 1648-1656, 2008. 
[6] D. K. Shukla, B. Keehn, and R. A. Müller, "Regional homogeneity of fMRI time series in autism spectrum disorders," Neuroscience Letters, vol. 476, no. 1, pp. 46-51, 2010.

[7] Z. Yao, L. Wang, Q. Lu, H. Liu, and G. Teng, "Regional homogeneity in depression and its relationship with separate depressive symptom clusters: a resting-state fMRI study," Journal of Affective Disorders, vol. 115, no. 3, pp. 430-438, 2009.

[8] W. B. Guo, X. L. Sun, L. Liu et al., "Disrupted regional homogeneity in treatment-resistant depression: a resting-state fMRI study," Progress in Neuro-Psychopharmacology and Biological Psychiatry, vol. 35, no. 5, pp. 1297-1302, 2011.

[9] Q. Z. Wu, D. M. Li, W. H. Kuang et al., "Abnormal regional spontaneous neural activity in treatment-refractory depression revealed by resting-state fMRI," Human Brain Mapping, vol. 32, no. 8, pp. 1290-1299, 2011.

[10] Z. Liu, C. Xu, Y. Xu et al., "Decreased regional homogeneity in insula and cerebellum: a resting-state fMRI study in patients with major depression and subjects at high risk for major depression," Psychiatry Research, vol. 182, no. 3, pp. 211-215, 2010.

[11] D. H. Peng, K. D. Jiang, Y. R. Fang et al., "Decreased regional homogeneity in major depression as revealed by resting-state functional magnetic resonance imaging," Chinese Medical Journal, vol. 124, no. 3, pp. 369-373, 2011.

[12] J. D. Chen, F. Liu, G. L. Xun et al., "Early and late onset, firstepisode, treatment-naive depression: same clinical symptoms, different regional neural activities," Journal of Affective Disorders, vol. 143, no. 1-3, pp. 56-63, 2012.

[13] B. D. Argall, Z. S. Saad, and M. S. Beauchamp, "Simplified intersubject averaging on the cortical surface using SUMA," Human Brain Mapping, vol. 27, no. 1, pp. 14-27, 2006.

[14] B. Fischl, M. I. Sereno, R. B. Tootell, and A. M. Dale, "Highresolution intersubject averaging and a coordinate system for the cortical surface," Human Brain Mapping, vol. 8, no. 4, pp. 272-284, 1999.

[15] B. Fischl, N. Rajendran, E. Busa et al., "Cortical folding patterns and predicting cytoarchitecture," Cerebral Cortex, vol. 18, no. 8, pp. 1973-1980, 2008.

[16] X. N. Zuo, T. Xu, L. Jiang et al., "Toward reliable characterization of functional homogeneity in the human brain: preprocessing, scan duration, imaging resolution and computational space," Neuroimage, vol. 65, pp. 374-386, 2013.

[17] M. B. First, R. L. Spitzer, and M. Gibbon, Structured Clinical Interview for DSM-IV Axis 1 Disorder, Biometrics Research Department, New York State Psychiatric Institute, New York, NY, USA, 1995.

[18] X. X. Xing, Y. L. Zhou, J. S. Adelstein, and X. N. Zuo, "PDEbased spatial smoothing: a practical demonstration of impacts on MRI brain extraction, tissue segmentation and registration," Magnetic Resonance Imaging, vol. 29, no. 5, pp. 731-738, 2011.

[19] X. N. Zuo and X. X. Xing, "Effects of non-local diffusion on structural MRI preprocessing and default network mapping: statistical comparisons with isotropic/anisotropic diffusion," PLoS ONE, vol. 6, no. 10, Article ID e26703, 2011.

[20] C. G. Yan, R. C. Craddock, X. N. Zuo, Y. F. Zang, and M. P. Milham, "Standardizing the intrinsic brain: towards robust measurement of inter-individual variation in 1000 functional connectomes," Neuroimage, vol. 80, pp. 246-262, 2013.

[21] D. N. Greve and B. Fischl, "Accurate and robust brain image alignment using boundary-based registration," NeuroImage, vol. 48 , no. 1 , pp. 63-72, 2009.
[22] J. L. Bernal-Rusiel, M. Atienza, and J. L. Cantero, "Determining the optimal level of smoothing in cortical thickness analysis: a hierarchical approach based on sequential statistical thresholding," NeuroImage, vol. 52, no. 1, pp. 158-171, 2010.

[23] S. Kuhn and J. Gallinat, "Resting-state brain activity in schizophrenia and major depression: a quantitative metaanalysis," Schizophrenia Bulletin, vol. 39, no. 2, pp. 358-365, 2013.

[24] M. M. Mesulam and E. J. Mufson, "Insula of the old world monkey. I. Architectonics in the insulo-orbito-temporal component of the paralimbic brain," Journal of Comparative Neurology, vol. 212, no. 1, pp. 1-22, 1982.

[25] E. J. Mufson and M. M. Mesulam, "Insula of the old world monkey. II. Afferent cortical input and comments on the claustrum," Journal of Comparative Neurology, vol. 212, no. 1, pp. 23-37, 1982.

[26] J. R. Augustine, "Circuitry and functional aspects of the insular lobe in primates including humans," Brain Research Reviews, vol. 22, no. 3, pp. 229-244, 1996.

[27] B. T. Lee, C. Seong Whi, K. Hyung Soo et al., "The neural substrates of affective processing toward positive and negative affective pictures in patients with major depressive disorder," Progress in Neuro-Psychopharmacology and Biological Psychiatry, vol. 31, no. 7, pp. 1487-1492, 2007.

[28] J. D. Townsend, N. K. Eberhart, S. Y. Bookheimer et al., "FMRI activation in the amygdala and the orbitofrontal cortex in unmedicated subjects with major depressive disorder," Psychiatry Research, vol. 183, no. 3, pp. 209-217, 2010.

[29] M. W. Schlund, G. Verduzco, M. F. Cataldo, and R. Hoehn-Saric, "Generalized anxiety modulates frontal and limbic activation in major depression," Behavioral and Brain Functions, vol. 8, article 8, 2012.

[30] I. M. Veer, C. F. Beckmann, M. J. van Tol et al., "Whole brain resting-state analysis reveals decreased functional connectivity in major depression," Frontiers in Systems Neuroscience, vol. 4, article 41, 2010.

[31] K. R. Cullen, D. G. Gee, B. Klimes-Dougan et al., "A preliminary study of functional connectivity in comorbid adolescent depression," Neuroscience Letters, vol. 460, no. 3, pp. 227-231, 2009.

[32] S. Lui, Q. Wu, L. Qiu et al., "Resting-state functional connectivity in treatment-resistant depression," The American Journal of Psychiatry, vol. 168, no. 6, pp. 642-648, 2011.

[33] H. Kober, L. F. Barrett, J. Joseph, E. Bliss-Moreau, K. Lindquist, and T. D. Wager, "Functional grouping and cortical-subcortical interactions in emotion: a meta-analysis of neuroimaging studies," NeuroImage, vol. 42, no. 2, pp. 998-1031, 2008.

[34] A. Stuhrmann, T. Suslow, and U. Dannlowski, "Facial emotion processing in major depression: a systematic review of neuroimaging findings," Biology of Mood \& Anxiety Disorders, vol. 1, no. 1, article 10, 2011.

[35] P. B. Fitzgerald, A. R. Laird, J. Maller, and Z. J. Daskalakis, "A meta-analytic study of changes in brain activation in depression," Human Brain Mapping, vol. 29, no. 6, pp. 683-695, 2008.

[36] D. Sliz and S. Hayley, "Major depressive disorder and alterations in insular cortical activity: a review of current functional magnetic imaging research," Frontiers in Human Neuroscience, vol. 6, article 323, 2012.

[37] T. Takahashi, M. Yücel, V. Lorenzetti et al., "Volumetric MRI study of the insular cortex in individuals with current and past major depression," Journal of Affective Disorders, vol. 121, no. 3, pp. 231-238, 2010. 
[38] H. Järnum, S. F. Eskildsen, E. G. Steffensen et al., "Longitudinal MRI study of cortical thickness, perfusion, and metabolite levels in major depressive disorder," Acta Psychiatrica Scandinavica, vol. 124, no. 6, pp. 435-446, 2011.

[39] P. Fusar-Poli, S. Borgwardt, A. Crescini et al., "Neuroanatomy of vulnerability to psychosis: a voxel-based meta-analysis," Neuroscience and Biobehavioral Reviews, vol. 35, no. 5, pp. 11751185, 2011.

[40] X. Liu, Y. Wang, H. Liu, Z. Liu, and W. Zhou, "Diffusion tensor imaging and resting state functional magnetic resonance imaging on young patients with major depressive disorder," Journal of Central South University, vol. 35, no. 1, pp. 25-31, 2010.

[41] D. M. Cannon, M. Ichise, D. Rollis et al., "Elevated serotonin transporter binding in major depressive disorder assessed using positron emission tomography and [11C]DASB, comparison with bipolar disorder," Biological Psychiatry, vol. 62, no. 8, pp. 870-877, 2007.

[42] A. Deschwanden, B. Karolewicz, A. M. Feyissa et al., "Reduced metabotropic glutamate receptor 5 density in major depression determined by [11C]ABP688 PET and postmortem study," The American Journal of Psychiatry, vol. 168, no. 7, pp. 727-734, 2011.

[43] H. Hanada, J. Imanaga, A. Yoshiiwa et al., "The value of ethyl cysteinate dimer single photon emission computed tomography in predicting antidepressant treatment response in patients with major depression," International Journal of Geriatric Psychiatry, vol. 28, no. 7, pp. 756-765, 2013.

[44] F. Liu, M. Hu, S. Wang et al., "Abnormal regional spontaneous neural activity in first-episode, treatment-naive patients with late-life depression: a resting-state fMRI study," Progress in Neuro-Psychopharmacology \& Biological Psychiatry, vol. 39, no. 2, pp. 326-331, 2012.

[45] R. Sprengelmeyer, J. D. Steele, B. Mwangi et al., "The insular cortex and the neuroanatomy of major depression," Journal of Affective Disorders, vol. 133, no. 1-2, pp. 120-127, 2011.

[46] W. C. Drevets, "Functional neuroimaging studies of depression: the anatomy of melancholia," Annual Review of Medicine, vol. 49, pp. 341-361, 1998.

[47] H. S. Mayberg, "Limbic-cortical dysregulation: a proposed model of depression," Journal of Neuropsychiatry and Clinical Neurosciences, vol. 9, no. 3, pp. 471-481, 1997. 

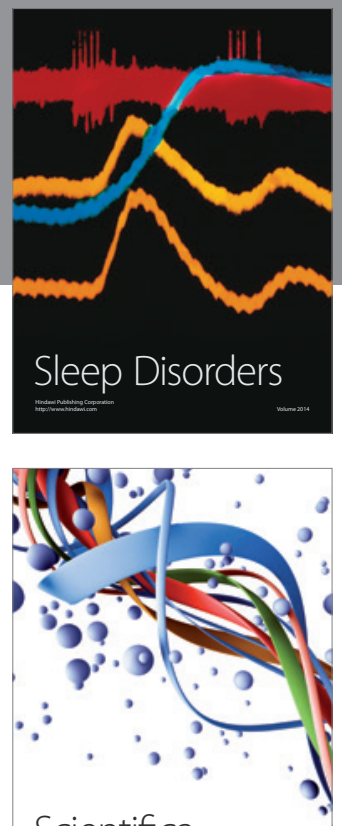

Scientifica
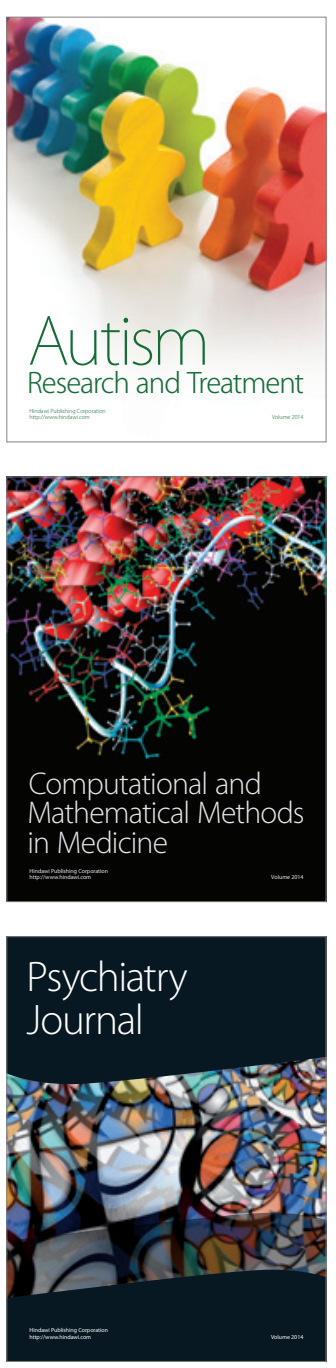
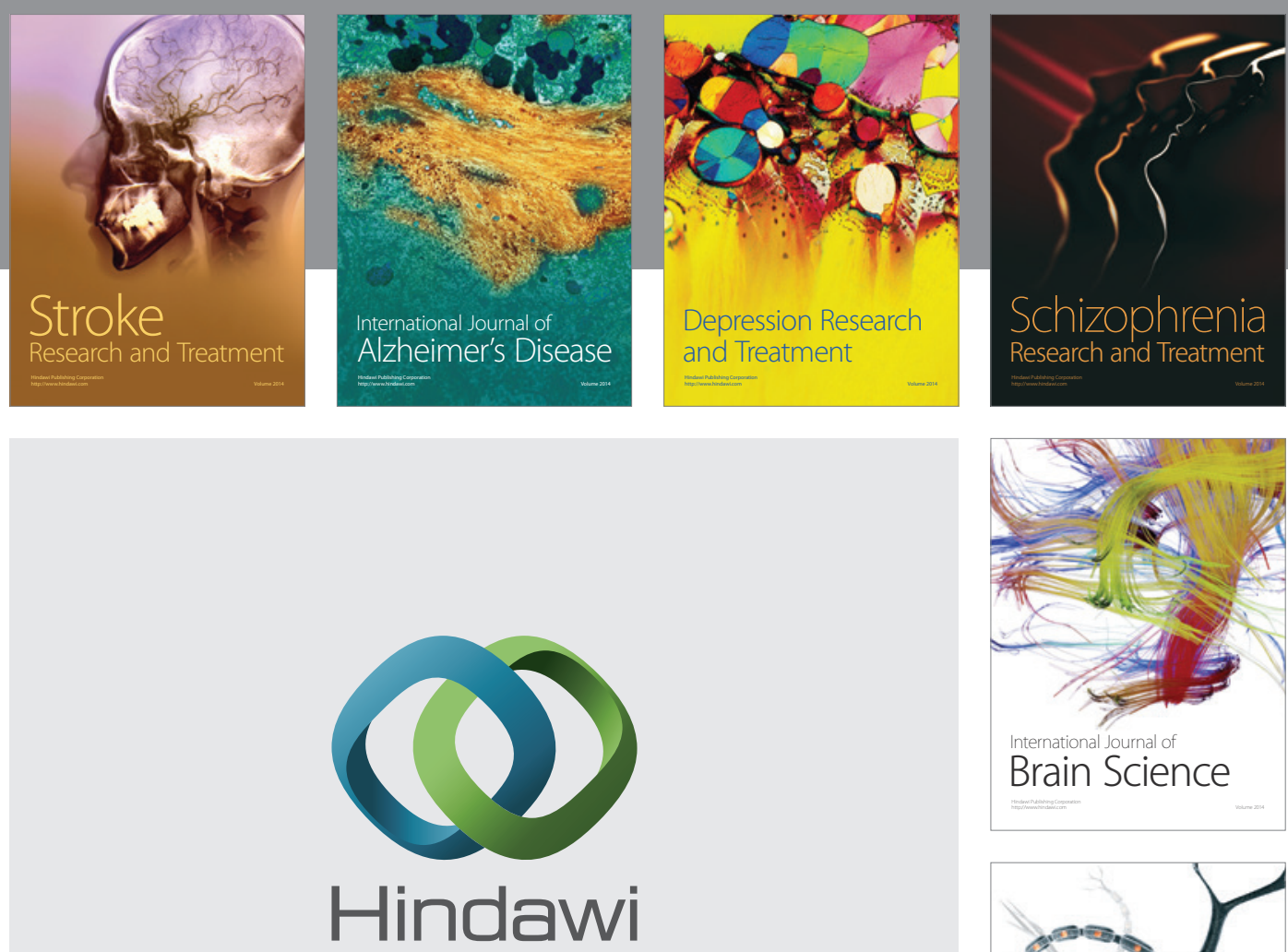

Submit your manuscripts at

http://www.hindawi.com
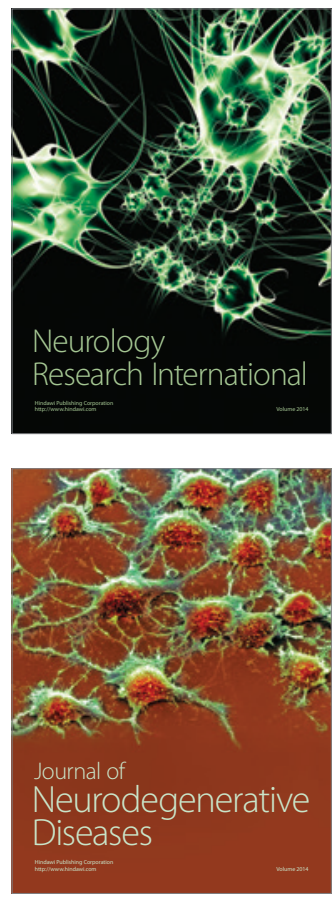

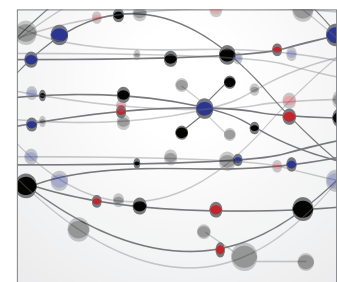

The Scientific World Journal
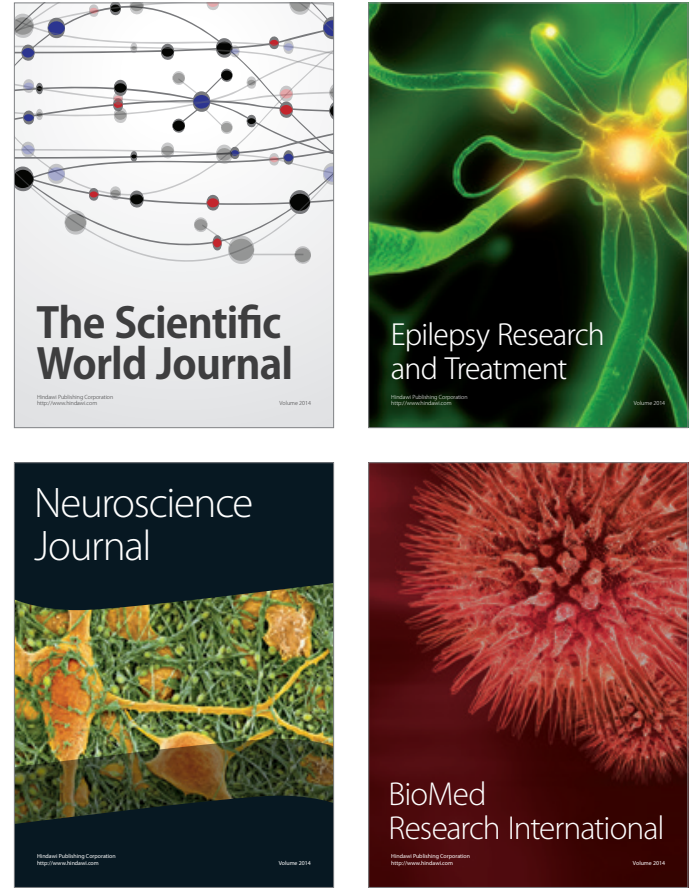

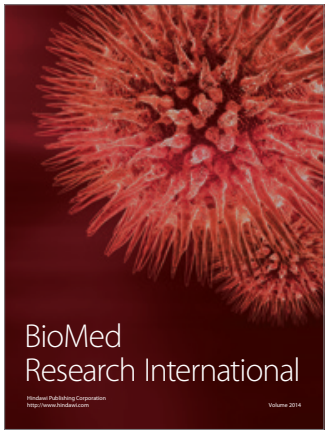

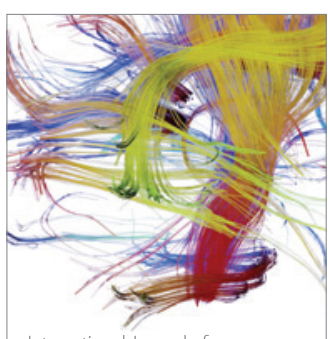

Brain Science

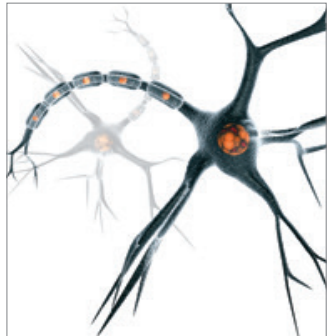

Neural Plasticity
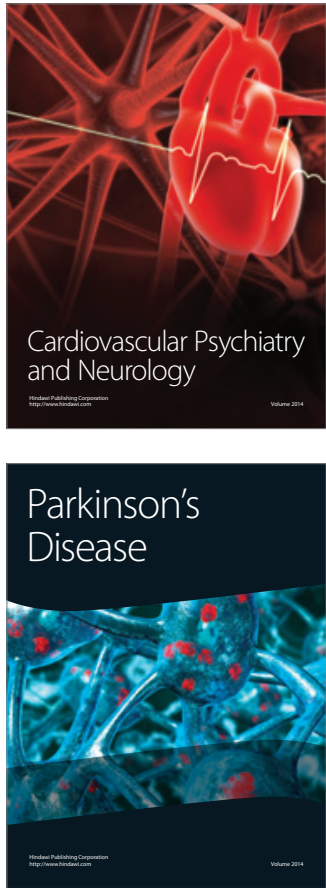